Ilchmann, Achim :

Robust Adaptive Stabilization of Discrete-time First-order Systems

Zuerst erschienen in:

Automatica 27 (1991), Nr. 5, S. 829-835

DOI: 10.1016/0005-1098(91)90037-3 


\title{
Robust Adaptive Stabilization of Discrete-time First-order Systems*
}

\author{
ACHIM ILCHMANN† \\ Key Words-Adaptive control; stabilizers; robust control; discrete-time systems. \\ An adaptive controller for a class of single-input single-output first-order \\ discrete-time systems which is robust with respect to certain nonlinear state, \\ input and output perturbations.
}

\begin{abstract}
An algorithm for the adaptive stabilization of single-input single-output time-varying first-order discretetime systems is presented. Only the knowledge of the sign of the feedback gain and an upper bound of it is required. The adaptive control law is robust with respect to nonlinear additive time-varying state, input and output perturbations. If the sign of the feedback gain is not known, it is shown that a simple modification of the control law leads to a controller which produces an asymptotically stable closed-loop system.
\end{abstract}

\section{INTRODUCTION}

THIS PAPER addresses the problem of adaptively stabilizing first-order, single-input single-output, linear systems of the form

$$
\left.\begin{array}{rl}
x_{t+1} & =a_{t} x_{t}+b_{t} u_{t} \\
y_{t} & =c_{t} x_{t}, \quad t \in \mathbb{N}
\end{array}\right\}
$$

where $a_{t}, b_{t}, c_{t} \in \mathbb{R}$ are unknown, only bounds of $a_{t}$ and of the feedback gain $b_{t} c_{t}$ are known. For different subclasses $\Sigma$ of (1.1) we present different controllers

$$
\begin{aligned}
k & : \mathbb{R} \times \mathbb{R} \times \mathbb{R} \\
\quad\left(y_{t}, y_{t+1}, k_{t}\right) & \rightarrow k_{t+1}
\end{aligned}
$$

so that the control law

$$
u_{t}=k_{t} y_{t}
$$

applied to a system $\left(a_{t}, b_{t}, c_{t}\right) \in \Sigma$ produces an exponentially or asymptotically decaying solution of the closed-loop system

$$
x_{t+1}=\left[a_{t}+k_{t} b_{t} c_{t}\right] x_{t} .
$$

The algorithms (1.2) are not based on identification or model reference approaches.

\footnotetext{
*Received 7 November 1989; revised 26 June 1990; received in final form 22 December 1990 . The original version of this paper was not presented at any IFAC meeting. This paper was recommended for publication in revised form by Associate Editor R. R. Bitmead under the direction of Editor P. C. Parks.

†Institut für Angewandte Mathematik der Universität Hamburg, Bundesstraße 55, D-2000 Hamburg 13, Germany.
}

Here, the controller "learns" from the measurements $y_{t}$ and adjusts the parameters in the closed loop system. Note that the parameter adjustment law (1.2) has only a "short" memory; at time $t+1$ the controller $k_{t+1}$ is adjusted by the knowledge of $y_{t+1}$ and $y_{t}$.

In the last five years, considerable progress has been made in the development of adaptive stabilization of continuous-time linear systems concerning the approach described in the previous paragraph. However, only a few authors tackled analogous questions and problems for discrete-time linear systems. Mudgett and Morse (1985) considered the class of time-invariant systems of the form (1.1) where only knowledge of an upper bound of $|b c|$ is required. It is not obvious how their controller can be simplified if the sign of $b c$ is known. Mårtensson (1986) presents a controller which stabilizes the class of time-invariant systems of the form (1.1) where neither the sign of $b c$ nor an upper bound of $|b c|$ need to be known. However, robustness results are not tackled and the proposed controller is very complicated compared to (2.2) or (5.2). Wang and Ljung (1989) considered a class of linear, single-input single-output systems of unknown order and allow certain perturbations. However, their algorithm ensures only bounded output signals.

In Section 2, a simple controller is presented which adaptively stabilizes each time-invariant system of the form (1.1). In Section 3, it is shown that this controller also works for certain time-varying systems of the form (1.1). In Section 4 , it is proved that the controller of Section 2 is robust with respect to certain time-varying nonlinear state, input and output perturbations. In Section 5, again time-invariant systems of the form (1.1) are considered. However, the known sign condition is weakened 
to $0<|b c|<g$. To produce an asymptotically decaying output, the controller of Section 2 needs only a small modification and is much simpler than the one introduced by Mudgett and Morse (1985). These results are illustrated by some numerical simulations.

2. THE TIME-INVARIANT KNOWN SIGN CASE

In this section, we consider the following class of linear time-invariant, first-order, single-input single-output systems

$$
\left.\begin{array}{l}
x_{t+1}=a x_{t}+b u_{t}, \quad t \in \mathbb{N} \\
y_{t}=c x_{t} \\
0<b c<g
\end{array}\right\}
$$

where $a, b, c \in \mathbb{R}$ are unknown. Only an upper bound $g>0$ for the feedback gain is required.

Proposition 1. Given a system $(a, b, c)$ of the form (2.1) and initial data $x_{0}, k_{0} \in \mathbb{R}$. Then the adaptive control law

$$
\begin{aligned}
u_{t} & =k_{t} y_{t} \\
k_{t+1} & :=k_{t}-\frac{1}{g} \frac{\operatorname{sign}\left(y_{t}\right) y_{t+1}}{1+\left|y_{t}\right|}
\end{aligned}
$$

produces an exponentially decaying solution of the closed-loop system (1.3).

Proof. Using the transformation

$$
\bar{k}_{t}:=k_{t}+\frac{a}{b c}
$$

(1.3) is equivalent to

$$
x_{t+1}=b c \bar{k}_{t} x_{t}
$$

To prove exponential stability of (2.4), assume that $x_{t} \neq 0$ and $\bar{k}_{t} \neq 0$ for all $t \in \mathbb{N}$, otherwise the proof would be complete. Let

$$
f_{t+1}:=\frac{1}{g} \frac{\operatorname{sign}\left(y_{t}\right) y_{t+1}}{1+\left|y_{t}\right|} .
$$

It follows from (2.2) and (2.3) that

$$
\bar{k}_{t+1}=\bar{k}_{t}-f_{t+1}=\left[1-\frac{f_{t+1}}{\bar{k}_{t}}\right] \bar{k}_{t} .
$$

By (2.4) and (2.5) we conclude

$$
0<\frac{f_{t+1}}{\bar{k}_{t}}=\frac{b c}{g} \frac{1}{1+\left|y_{t}\right|^{-1}}<\frac{b c}{g}<1
$$

and hence

$$
0<1-\frac{b c}{g}<1-\frac{f_{t+1}}{\vec{k}_{t}}<1 .
$$

It follows from (2.6) that

$$
\bar{k}_{t+1}=\prod_{i=0}^{t}\left(1-\frac{f_{i+1}}{\bar{k}_{i}}\right) \bar{k}_{0}
$$

and therefore (2.8) implies that $\bar{k}_{t}$ is monotonically decreasing or increasing. Thus, there exists some $\bar{k}_{\infty} \geq 0$ so that

$$
\lim _{t \rightarrow \infty} \bar{k}_{t}=\bar{k}_{\infty}
$$

If $\bar{k}_{\infty}=0$, then for every $\omega \in(0,1)$ there exists some $M>0$ such that

$$
\left|x_{t+1}\right| \leq M \omega^{t}\left|x_{0}\right| \text { for all } t \in \mathbb{N} .
$$

If $\bar{k}_{\infty}>0$, then it follows from (2.9) that

$$
\lim _{t \rightarrow \infty}\left|1-\frac{f_{t+1}}{\bar{k}_{t}}\right|=1 \text {. }
$$

Hence, by (2.8),

$$
\lim _{t \rightarrow \infty} \frac{f_{t+1}}{\bar{k}_{t}}=0
$$

and therefore

This yields

$$
\lim _{t \rightarrow \infty} 1+\frac{1}{\left|y_{t}\right|}=\infty
$$

$$
\lim _{t \rightarrow \infty} x_{t}=0 .
$$

Since $\left|\bar{k}_{t}\right|$ is monotonically decreasing, we obtain from (2.12) and (2.4) that there exists some $\omega \in(0,1)$ and $t_{0} \in \mathbb{N}$ so that

$$
\left|b c \bar{k}_{t}\right| \leq \omega<1 \text { for all } t \geq t_{0} .
$$

This implies (2.11) and the proof is complete.

Remark 1. The controller (2.2) can be modified by

$$
k_{t+1}:=k_{t}-\frac{1}{g} \frac{\operatorname{sign}\left(y_{t}\right) y_{t+1}}{\left(\alpha+\left|y_{t}\right|^{q}\right)^{1 / p}}
$$

where $\alpha>0,0<p \leq q<\infty$. We prefer the presentation (2.2) for technical reasons. This does not effect the theoretical results of the present paper and all propositions remain valid. However, we do not prove-but computer simulations have shown-that $p=q=2$ yields better results than $p=q=1$ in the following sense: There is nearly no difference comparing the speed of convergence, but for $p=q=2,\left|y_{t}\right|$ does not increase so high before it settles to zero.

Remark 2. Suppose $\left|y_{t}\right|$ is large. Then by (2.6) $-(2.8), \quad \bar{k}_{t+1} \approx(1-(b c / g)) \bar{k}_{t}$ and $\bar{k}_{t+1}$ is decreasing almost as fast as possible. The convergence becomes faster if the bound $g$ is tight. For $\left|y_{t}\right|$ small, the opposite statement holds true. If the initial value $x_{0}$ is small and $k_{0}$ is so that $\left|a+k_{0} b c\right| \gg 1$, then the convergence of $k_{t}$ is slow. This has the effect that the output becomes very large before $t \in \mathbb{N}$ is reached such that $\left|a+k_{t} b c\right|<1$ and the solution starts to settle to zero. See Figs 1, 2 and 3 for illustration. 


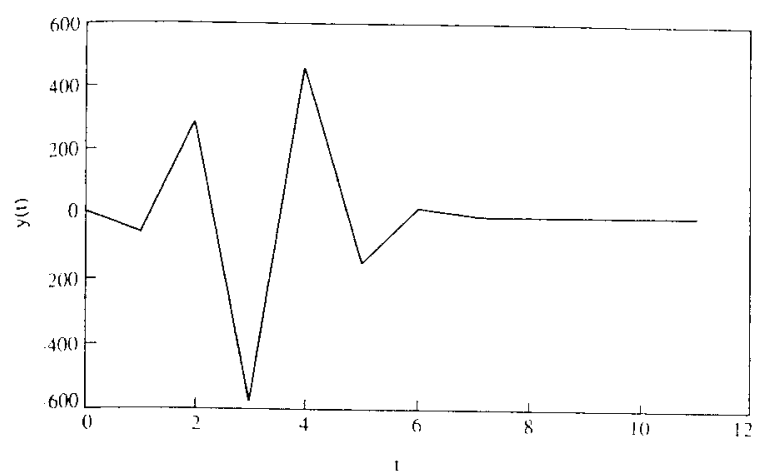

FIG. 1. Output of system (2.1) using adaptive control law (2.2). $a=-10, b=1, c=6, g=10, x(0)=1, k(0)=0$.

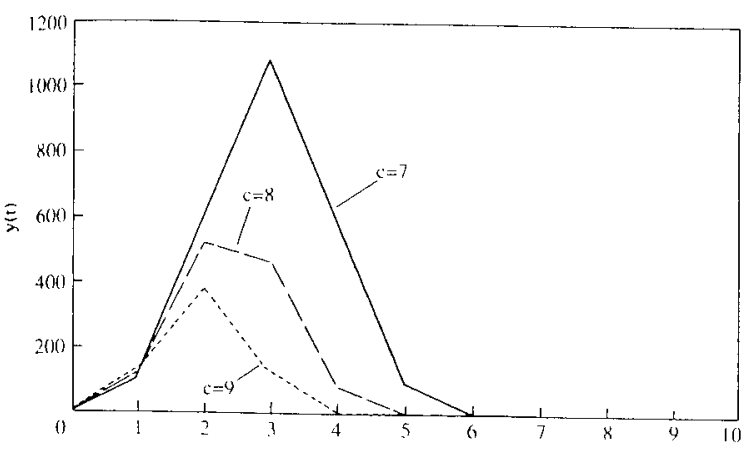

FIG. 2. Output of system (2.1) using adaptive control law (2.2). $a=15, b=1, x(0)=1, k(0)=0$.

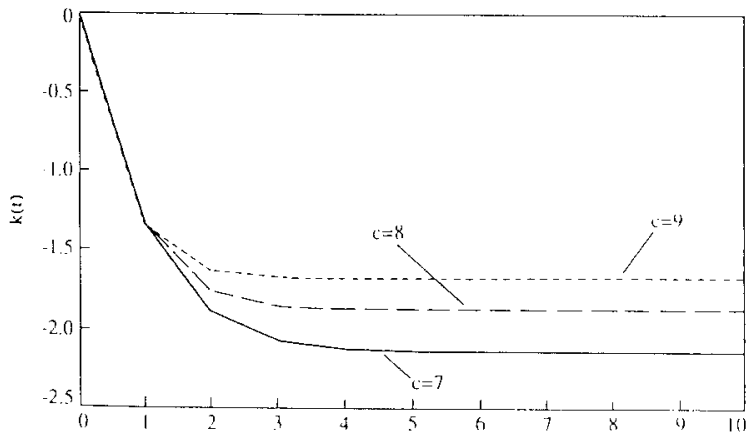

Fig. 3. Adaptive feedback gain of system (2.1) in adaptive control law (2.2). $a=15, b=1, x(0)=1, k(0)=0$.

\section{THE TIME-VARYING KNOWN SIGN CASE}

In this section, the class (2.1) is enlarged by allowing certain time-varying additive perturbations of $a, b$ and $c$. We consider the following class

$$
\left.\begin{array}{l}
x_{t+1}=\left(a+a_{t}\right) x_{t}+\left(b+b_{t}\right) u_{t} \\
y_{t}=\left(c+c_{t}\right) x_{t} \\
\left|a_{t}\right| \leq \alpha,\left|b_{t}\right| \leq \beta,\left|c_{t}\right| \leq \gamma \\
0<b c<g
\end{array}\right\}
$$

where $a, a_{t}, b, b_{t}, c, c_{t} \in \mathbb{R}$ are unknown, $\alpha, \beta, \gamma \geq 0$ and $g>0$ are given. However, for the class (3.1) we can only expect the weaker result, that the solution of the closed-loop system (1.3) tends asymptotically, instead of exponentially, to zero as $t \rightarrow \infty$. First, we consider the case that time variance occurs only in $a$.

Proposition 2. Suppose $\left(a+a_{t}, b, c\right)$ is a system of the class (3.1) with $\alpha<\frac{1}{4}, \beta=\gamma=0$ and initial data $x_{0}, k_{0} \in \mathbb{B}$. Then the adaptive control law (2.2) produces an asymptotically stable solution of the closed-loop system

$$
x_{t+1}=\left[a+a_{t}+k_{t} b c\right] x_{t}, \quad t \in \mathbb{N} .
$$

Proof. Using the transformation (2.3), it remains to show that the solution $x_{t}$ of

$$
x_{t+1}=\left[a_{t}+\bar{k}_{t} b c\right] x_{t}
$$

tends to zero as $t \rightarrow \infty$. Without restriction of generality assume that $x_{t} \neq 0$ for all $t \in \mathbb{N}$. Since

$$
\frac{f_{t+1}}{\bar{k}_{t}}=\frac{c b}{g} \frac{1}{1+\left|y_{t}\right|^{-1}} \frac{y_{t+1}}{y_{t+1}-a_{t} y_{t}}
$$

we obtain

$$
\bar{k}_{t+1}=\bar{k}_{t}\left[1-\frac{b c}{g} \frac{1}{1+\left|y_{t}\right|^{-1}}\right]-\frac{a_{t}}{g} \frac{1}{\sqrt{1+y^{-2}}}
$$

resp.

$$
g\left(\bar{k}_{t}-\bar{k}_{t+1}\right)=\frac{1}{1+\left|y_{t}\right|^{-1}}\left[\bar{k}_{t} b c+a_{t}\right] .
$$

Suppose the evolution of $\bar{k}_{t}$ satisfies the following rules:

$$
\begin{gathered}
\left|\bar{k}_{t+1}\right|<\frac{3 \alpha}{b c} \quad \text { if } \quad\left|\bar{k}_{t}\right|<\frac{\alpha}{b c} \\
-\frac{\alpha}{b c}<\bar{k}_{t+1}<\bar{k}_{t} \quad \text { if } \quad \bar{k}_{t} \geq \frac{\alpha}{b c} \\
\bar{k}_{t}<\bar{k}_{t+1}<\frac{\alpha}{b c} \quad \text { if } \quad \bar{k}_{t} \leq-\frac{\alpha}{b c} .
\end{gathered}
$$

We proceed in several steps.

(i) If there exist a $t_{0} \in \mathbb{N}$ so that $\left|\bar{k}_{t_{0}}\right|<\alpha / b c$, it follows from (3.6)-(3.8) that $\left|\bar{k}_{t}\right|<3 \alpha / b c$ for all $t>t_{0}$ and thus

$$
\left|a_{t}+\bar{k}_{t} b c\right|<4 \alpha \text { for all } t>t_{0} .
$$

Hence (3.3) is exponentially stable since $\alpha<\frac{1}{4}$.

(ii) As long as $\left|\bar{k}_{t}\right| \geq(\alpha / b c)$, it follows from (3.6)-(3.8) that $\bar{k}_{t}$ is monotonically decreasing (resp. increasing) if $\bar{k}_{0} \geq \alpha / b c$ (resp. $\bar{k}_{0} \leq \alpha / b c$ ). Therefore $\lim \bar{k}_{t}$ exists and we put

$$
\lim _{t \rightarrow \infty} \bar{k}_{t}=\bar{k}_{\infty}
$$

(3.10) applied to (3.5) gives

$$
\lim _{t \rightarrow \infty} \frac{1}{1+\left|y_{t}\right|^{-1}}\left[\bar{k}_{x} b c+a_{t}\right]=0 .
$$


Then either

$$
\lim _{t \rightarrow \infty} \frac{1}{1+\left|y_{t}\right|^{-1}}=0
$$

or

$$
\lim _{t \rightarrow \infty} \bar{k}_{x} b c+a_{t}=0 .
$$

If (3.13) holds true, then exponential stability of (3.3) is obvious. If (3.12) holds true, then $\lim \left|y_{t}\right|=0$, whence $\lim \left|x_{t}\right|=0$. Thus it remains to prove $(3.6)-(3.8)$.

(iii) Suppose $\left|\bar{k}_{t}\right|<\alpha / b c$ for some $t \in \mathbb{N}$. We obtain by $(2.5)$

$$
\left|f_{t+1}\right|=\frac{1}{g} \frac{\left|c\left(a_{t}+b c \bar{k}_{t}\right) x_{t}\right|}{1+\left|y_{t}\right|}<\frac{1}{g}\left|a_{t}+b c \bar{k}_{t}\right|<\frac{2 \alpha}{b c} .
$$

Therefore

$$
\left|\bar{k}_{t+1}\right| \leq\left|\bar{k}_{t}\right|+\left|f_{t+1}\right|<\frac{3 \alpha}{b c}
$$

which proves (3.6). To prove (3.7), first assume that $a_{t} \geq 0$. Then (3.5) implies that $\vec{k}_{t+1}<\bar{k}_{t}$. Since

$$
\frac{g}{b c} \alpha>\alpha>a_{t}>a_{t} \frac{1}{1+\left|y_{t}\right|^{-1}},
$$

we obtain

$$
-\frac{\alpha}{b c}<-\frac{a_{t}}{g} \frac{1}{1+\left|y_{t}\right|^{-1}}
$$

whence

$$
-\frac{\alpha}{b c}<\bar{k}_{t}\left[1-\frac{b c}{g} \frac{1}{1+\left|y_{t}\right|^{-1}}\right]-\frac{a_{t}}{g} \frac{1}{1+\left|y_{t}\right|^{-1}}=\bar{k}_{t+1} .
$$

This proves (3.7) for the case $a_{t} \geq 0$. If $a_{t}<0$, then $\bar{k}_{t+1}$ is positive, and hence $-(\alpha / b c)<\bar{k}_{t+1}$. Since $\vec{k}_{t}>\alpha / b c$,

$$
-\bar{k}_{t} \frac{b c}{g} \frac{1}{1+\left|y_{t}\right|^{-1}}-\frac{a_{t}}{g} \frac{1}{1+\left|y_{t}\right|^{-1}}<0
$$

and therefore, by (3.5), $\bar{k}_{t+1}<\bar{k}_{t}$. Thus, (3.7) is shown. The proof of (3.8) uses similar arguments; it is omitted for brevity.

The following proposition shows that the adaptive control law (2.2) is capable of tolerating time-varying additive perturbations of $a, b$ and $c$.

Proposition 3. Let $\left(a+a_{t}, b+b_{t}, c+c_{t}\right)$ be a system of the class (3.1) with initial data $x_{0}$, $k_{0} \in \mathbb{R}$. Then the adaptive control law (2.2) produces an asymptotically stable output of the closed-loop system

$$
x_{t+1}=\left[a+a_{t}+k_{t}\left(b c+\delta_{t}\right)\right] x_{t}, \quad t \in \mathbb{N}
$$

where

$$
\delta_{t}:=b c_{t}+b_{t} c+b_{t} c_{t}
$$

provided the perturbations $a_{t}, b_{t}$, and $c_{t}$ are small enough.

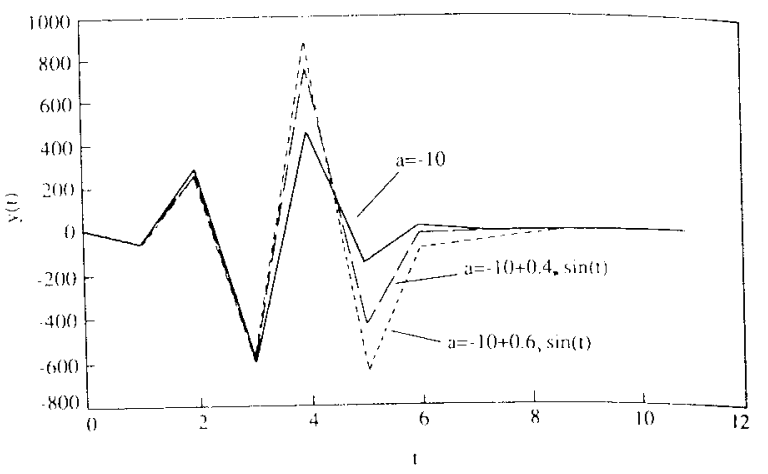

FIG. 4. Output of system 3.1 with time variations only in $a$. $b=1, c=6, g=10, x(0)=1, k(0)=0$.

Proof. The proof is similar to that of Proposition 2. Choose positive $\varepsilon$ and $\hat{\varepsilon}$ so that the perturbations satisfy the following inequalities

$$
\left.\begin{array}{l}
\left|\delta_{t}\right|<\hat{\varepsilon} \\
\left|a_{t}-\frac{a}{b c} \delta_{t}\right|<\varepsilon \\
0<b c-\hat{\varepsilon}<b c+\hat{\varepsilon}<g \\
1+2 \frac{b c+\hat{\varepsilon}}{b c-\hat{\varepsilon}}+\left(\frac{b c+\hat{\varepsilon}}{b c-\hat{\varepsilon}}\right)^{2}<\varepsilon^{-1}
\end{array}\right\} .
$$

It can be shown that this implies the existence of some positive $\alpha, \beta, \gamma>0$ so that

$$
\left|a_{t}\right| \leq \alpha, \quad\left|b_{t}\right| \leq \beta, \quad\left|\gamma_{t}\right| \leq \gamma
$$

is fulfilled for all $t \in \mathbb{N}$. The transformation $\bar{k}_{t}=k_{t}+a / b c$ leads to

$$
x_{t+1}=\left[\xi_{t}+\bar{k}_{t}\left(b c+\delta_{t}\right)\right] x_{t}
$$

where $\xi_{t}:=a_{t}-(a / b c) \delta_{t}$. By (2.5), (2.6) and (3.16) we conclude

$$
\bar{k}_{t+1}=\bar{k}_{t}\left[1-\frac{b c+\delta_{t}}{g} \frac{1}{1+\left|y_{t}\right|^{-1}}\right]-\frac{\xi_{t}}{g} \frac{1}{1+\left|y_{t}\right|^{-1}} .
$$

Similarly to the proof of (3.6)-(3.8) we can show

$$
\begin{array}{rlrl}
\left|\bar{k}_{t+1}\right|<\frac{\varepsilon(3 b c+\hat{\varepsilon})}{(b c-\hat{\varepsilon})^{2}} & \text { if } & \left|\bar{k}_{t}\right|<\frac{\varepsilon}{b c-\hat{\varepsilon}} \\
-\frac{\varepsilon}{b c-\hat{\varepsilon}}<\bar{k}_{t+1}<\bar{k}_{t} \quad & \text { if } \quad \bar{k}_{t} \geq \frac{\varepsilon}{b c-\hat{\varepsilon}} \\
\bar{k}_{t}<\bar{k}_{t+1}<\frac{\varepsilon}{b c-\hat{\varepsilon}} \quad & \text { if } & \bar{k}_{t} \leq-\frac{\varepsilon}{b c-\hat{\varepsilon}} .
\end{array}
$$

Now the remainder of the proof follows by exactly the same arguments to that in Proposition 2. See Fig. 4 for illustration. 4. ROBUSTNESS OF THE CONTROLLER WITH
RESPECT TO NONLINEAR PERTURBATION

The adaptive control law (2.2) is tolerant with respect to structural disturbances. "Structural" 
here means that the perturbations are linearly uniformly bounded.

Two types of perturbations are considered:

(i) $x_{t+1}=a x_{t}+d\left(t, x_{t}\right)+b u_{t}$

where the map $d: \mathbb{N} \times \mathbb{R} \rightarrow \mathbb{R}$ satisfies for some $d_{0}>0$

$$
|d(t, x)| \leq d_{0}|x| \quad \text { for all } t \in \mathbb{N}, \quad x \in \mathbb{R}
$$

i.e. time-varying nonlinear state dependent disturbances of uniformly bounded finite gain; and

(ii) $x_{t+1}=a x_{t}+b\left[u_{t}+h\left(t, x_{t}, u_{t}, y_{t}\right)\right]$ where the map $h: \mathbb{N} \times \mathbb{R}^{3} \rightarrow \mathbb{R}$ satisfies for some $h_{1}, h_{2}, h_{3}>0$

$$
\begin{aligned}
& \begin{aligned}
h(t, x, u, y)= & h_{1}(t) \cdot x+h_{2}(t) \cdot u \\
& +h_{3}(t) y
\end{aligned} \\
& \text { for all } x, u, y \in \mathbb{R}, \quad t \in \mathbb{N} \\
& \text { where }\left|h_{i}(t)\right| \leq \bar{h} \text { for all } t \in \mathbb{N}
\end{aligned}
$$

i.e. time-varying nonlinear state, input and output dependent perturbations of the plant input of bounded growth. Then the following robustness results hold true.

Proposition 4. Given a system $(a, b, c)$ of the form (2.1), a nonlinear map (4.1), and initial data $x_{0}, k_{0} \in \mathbb{R}$. Then for $d_{0}<\frac{1}{4}$ the adaptive control law (2.2) applied to the nonlinear system

$$
\begin{aligned}
x_{t+1} & =a x_{t}+d\left(t, x_{t}\right)+b u_{t} \\
y_{t} & =c x_{t}
\end{aligned}
$$

produces an asymptotically decaying solution of the closed-loop system

$$
x_{t+1}=\left[a+k_{t} b c\right] x_{t}+d\left(t, x_{t}\right) .
$$

Proof. The proof is similar to that of Proposition 2. Therefore, only the essential steps are presented. Put $d_{0}=\alpha$. The transformation (2.3) yields

$$
x_{t+1}=\bar{k}_{t} b c x_{t}+d\left(t, x_{i}\right) .
$$

Assume $x_{t} \neq 0$ for all $t \in N$. Then

$$
g\left(\bar{k}_{t}-\bar{k}_{t+1}\right)=\bar{k}_{t} \frac{b c\left|y_{t}\right|}{1+\left|y_{t}\right|}-\frac{c \cdot d\left(t, x_{t}\right)}{1+\left|y_{t}\right|} .
$$

Suppose (3.6)-(3.8) hold true:

(i) If there exists a $t_{0} \in \mathbb{N}$ so that $\left|\bar{k}_{t_{0}}\right|<\alpha / b c$, then it follows from (4.5) and (4.1) that the solution of (4.5) resp. (4.4) is exponentially decaying.

(ii) if $\bar{k}_{t} \geq \alpha / b c$ for all $t \in \mathbb{N}$ then (3.10) is valid and (4.6) implies

$$
\lim _{t \rightarrow \infty} \frac{1}{1+\left|y_{t}\right|^{-1}}\left[b c \bar{k}_{t}-\frac{c \cdot d\left(t, x_{t}\right)}{\left|y_{t}\right|}\right]=0 .
$$

Since $\left|c \cdot d\left(t, x_{t}\right) / y_{t}\right| \leq d_{0}$ it is obvious that the solution of (4.5) tends to zero as $t$ tends to infinity. It remains to prove (3.6) $-(3.8)$.

(iii) If $\left|\bar{k}_{t}\right|<\alpha / b c$ then

$$
\left|f_{t+1}\right|=\frac{|c|}{g} \frac{\bar{k}_{t} b c x_{t}+d\left(t, x_{t}\right)}{1+\left|y_{t}\right|}
$$

and (4.1) give $\left|\bar{k}_{t+1}\right| \leq\left|\bar{k}_{t}\right|+\left|f_{t+1}\right|<3 \alpha / b c$. This proves (3.6). To prove (3.7), first assume that $c \cdot d\left(t, x_{t}\right) \geq 0$. Since

$$
\bar{k}_{t+1}=\bar{k}_{t}\left[1-\frac{b c}{g} \frac{1}{1+\left|y_{t}\right|^{-1}}\right]-\frac{c \cdot d\left(t, x_{t}\right)}{g \cdot\left(1+\left|y_{t}\right|\right)}
$$

we obtain $\bar{k}_{t+1}<\bar{k}_{\boldsymbol{t}}$. The inequality

$$
\begin{gathered}
\frac{g}{b c}>1>\frac{c \cdot d\left(t, x_{t}\right)}{d \cdot\left(1+\left|y_{t}\right|\right)} \text { yields } \\
-\frac{\alpha}{b c}<\bar{k}_{t}\left[1-\frac{b c}{g} \frac{1}{1+\left|y_{t}\right|^{-1}}\right]-\frac{c \cdot d\left(t, x_{t}\right)}{g \cdot\left(1+\left|y_{t}\right|\right)}=\bar{k}_{t+1} .
\end{gathered}
$$

This proves (3.7) for the case $c \cdot d\left(t, x_{t}\right) \geq 0$. The case $c \cdot d\left(t, x_{t}\right)<0$ is easy and therefore omitted. The proof of (3.8) uses similar arguments.

Proposition 5. Given a system $(a, b, c)$ of the form (2.1), nonlinear maps (4.1) and (4.2), and initial data $x_{0}, k_{0} \in \mathbb{R}$. Then there exists a sufficiently small number $\delta \geq \max \left\{d_{0}, \bar{h}\right\}>0$ such that the adaptive control law (2.2) applied to the nonlinear system

$$
\begin{aligned}
x_{t+1} & =a x_{t}+d\left(t, x_{t}\right)+b\left[u_{t}+h\left(t, x_{t}, u_{t}, y_{t}\right)\right] \\
y_{t} & =c x_{t}
\end{aligned}
$$

produces an asymptotically decaying solution of the closed-loop system

$$
\begin{aligned}
x_{t+1}= & {\left[a+b \cdot h_{1}(t)+c h_{3}(t)+k_{t} b c\left(h_{2}(t)+1\right)\right] } \\
& \times x_{t}+d\left(t, x_{t}\right) .
\end{aligned}
$$

Proof. Without restriction of generality suppose that $x_{t} \neq 0$ for all $t \in \mathbb{N}$. The transformation $\bar{k}_{t}=k_{t}+a / b c$ converts (4.8) to

$$
x_{t+1}=\left\{\bar{k}_{t}\left[b c+\delta_{t}\right]+\xi_{t}\right\} x_{t}+d\left(t, x_{t}\right)
$$

where

$$
\delta_{t}:=-b c h_{2}(t)
$$

and

$$
\xi_{t}:=b \cdot h_{1}(t)+c h_{3}(t)-a h_{2}(t) .
$$

Furthermore

$$
\bar{k}_{t+1}=\bar{k}_{t}\left[1-\frac{b c+\delta_{t}}{g} \frac{1}{1+\left|y_{t}\right|^{-1}}\right]-\frac{\psi\left(t, x_{t}\right)}{g \cdot\left(1+\left|y_{t}\right|\right)}
$$

where

$$
\psi\left(t, x_{t}\right):=\xi_{t} \cdot\left|y_{t}\right|-c \cdot d\left(t, x_{t}\right) .
$$


Choose $\delta, \alpha>0$ sufficiently small such that

$$
\left|\delta_{t}\right|<\bar{\delta}:=\min \left\{\frac{b c}{2}, g-b c\right\} \text { for all } t \in \mathbb{N}
$$

$$
\begin{gathered}
2 \alpha\left[\frac{g}{b c-\bar{\delta}}+1\right]<1 \\
0<\left|\xi_{t}\right|+d_{0}<\alpha .
\end{gathered}
$$

Then

$$
0<b c-\bar{\delta} \leq b c+\delta_{t}<g
$$

and

$$
0<\frac{\alpha}{b c+\delta_{t}} \leq \frac{\alpha}{b c-\bar{\delta}}=: \varepsilon .
$$

The proof proceeds similarly to that of Propositions 2 and 4, and the following inequalities can be shown; we omit it for brevity.

$$
\begin{aligned}
\left|\bar{k}_{t+1}\right|<2 \varepsilon+\frac{\alpha}{g} & \text { if } & \left|\bar{k}_{t}\right|<\varepsilon \\
-\varepsilon<\bar{k}_{t+1}<\bar{k}_{t} & \text { if } & \bar{k}_{t} \geq \varepsilon \\
\bar{k}_{t}<\bar{k}_{t+1}<\varepsilon & \text { if } & \bar{k}_{t} \leq \varepsilon .
\end{aligned}
$$

The remainder of the proof can be completed as in part (i) and (ii) of the proof of Proposition 2. Note that a rearrangement of (4.10) gives

$$
g\left(\bar{k}_{t}-\bar{k}_{t+1}\right)=\frac{1}{1+\left|y_{t}\right|^{-1}}\left[\bar{k}_{t} \frac{b c+\delta_{t}}{g}+\frac{\psi\left(t, x_{t}\right)}{\left|y_{t}\right|}\right] .
$$

\section{THE UNKNOWN SIGN CASE}

In this section, we weaken the sign condition $0<b c<g$ and consider the following class of systems.

$$
\left.\begin{array}{l}
x_{t+1}=a x_{t}+b u_{t} \\
y_{t}=c x_{t}, \quad t \in \mathbb{N} \\
0<|b c|<g
\end{array}\right\}
$$

where $a, b, c \in \mathbb{B}$ are unknown, $g>0$ is given.

The problem of adaptive stabilization of systems of the class (5.1) is much easier to solve than the analogous problem for continuous time systems. In the continuous case, one has to implement a switching function and a so-called Nussbaum gain into the feedback loop. Then the correct sign of the gain is found adaptively. However, it is not known in advance how many switchings are necessary or in which time the correct sign will be found out. See Morse (1983), Nussbaum (1983) and Willems and Byrnes (1984). For systems of the form (5.1) only two iterations are necessary to find out the correct sign $S \in\{1,-1\}$ of the control law $u_{t}=S k_{t} y_{t}$. In this sense the algorithm is much simpler than the one presented in Mudgett and Morse (1985).

Proposition 6. Suppose the system $(a, b, c)$ belongs to the class (5.1). Then for arbitrary initial data $x_{0}, k_{0} \in \mathbb{R}$, the control law

$$
u_{t}=S_{t} k_{t} y_{t}
$$

where $k_{t}$ is given by (2.2), $S_{0}=S_{1}=1$, and for $t>1$

$$
S_{t}:=\left\{\begin{array}{rll}
1 & \text { if } & \left\{y_{0} y_{2} / y_{1}^{2}<1 \text { or } y_{1}=0\right\} \\
-1 & \text { if } & y_{0} y_{2} / y_{1}^{2}>1
\end{array}\right.
$$

produces an exponentially decaying output of the closed-loop system

$$
x_{t+1}=\left[a+S_{t} k_{t} b c\right] x_{t} .
$$

Proof. Applying the transformation

$$
\vec{k}_{t}:=k_{t}+S_{t} \frac{a}{b c}
$$

to (5.4) yields

$$
x_{t+1}=S_{t} \bar{k}_{t} b c x_{t} .
$$

Then, if $y_{1} \neq 0$,

$$
\frac{y_{2} y_{0}}{y_{1}^{2}}=\frac{\bar{k}_{1}}{\bar{k}_{0}}
$$

Assume, for a moment, that the control strategy (2.2) is applied. If $b c>0$, it follows from (2.6) and (2.7) that $\operatorname{sign} \bar{k}_{t}=\operatorname{sign} \bar{k}_{t+1}$ and $\left|\bar{k}_{t+1}\right|=\left|\bar{k}_{t}\right|$. Therefore

$$
\bar{k}_{t+1} / \bar{k}_{t}<1 \quad \text { if } \quad b c>0
$$

If $b c<0$, we have

$$
-1<\frac{c b}{g}<\frac{c b}{g} \frac{1}{1+\left|y_{t}\right|^{-1}}=\frac{f_{t+1}}{\bar{k}_{t}}<0
$$

whence

$$
2>1-\frac{c b}{g}>1-\frac{f_{t+1}}{\bar{k}_{t}}>1 .
$$

This implies that $\operatorname{sign} \bar{k}_{t}=\operatorname{sign} \bar{k}_{t+1}$ and, by (2.6), $\left|\bar{k}_{t+1}\right|>\left|\bar{k}_{t}\right|$. Therefore

$$
\bar{k}_{t+1} / \bar{k}_{t}>1 \text { if } \quad b c<0 .
$$

Now (5.7) and (5.8) together with (5.6) yield that the switching function (5.3) ensures the correct sign modification of $b c$. Then the result follows from the proof of Proposition 1.

Remark 3. Obviously, the control law (5.2) does not stabilize the system if the system (5.1) is subjected to disturbances of the form (4.1) of (4.2). However, if the disturbances are of finite domain (i.e. nonzero for finite time) then the following

$$
S_{t}:=\left\{\begin{array}{rll}
1 & \text { if } & \left\{y_{t-1} y_{t+1} / y_{t}^{2}<1 \text { or } y_{t}=0\right\} \\
-1 & \text { if } & y_{t-1} y_{t+1} / y_{t}^{2}>1
\end{array}\right.
$$

stabilizes the system. 


\section{CONCLUSIONS}

The purpose of the present paper is to show the existence of a simple controller which stabilizes linear one-dimensional single-input single-output systems. The controller is simple in the sense that at the time $t$, the adjustment of $k_{t}$ only depends on the data of $y_{t}$ and $y_{t+1}$ and an $a$ priori known upper bound $g>0$ of the feedback gain $|b c|$. The controller presented here is of order two. However, there is no need for a memory for the complete past of $y(s), s \in[0, t]$, which was used in the continuous case, $k(t)=\int_{0}^{t} y(s)^{2} d s+k_{0}$ in Willems and Byrnes (1984). Furthermore, the controller is capable of tolerating time-varying nonlinear additive state, input and output perturbations. If the sign of the feedback gain $b c$ is not known, then the discrete-time case is much easier to handle than the continuous-time case. There is no need to implement a Nussbaum-type controller [see Willems and Byrnes (1984)]. However, as the simulations show, the output becomes very large before settling to zero even when the initial condition is very small. This is a problem which migh be solved by choosing the parameters $p$ and $q$ in the modified controller of (2.2) appropriately (see Remark 1). The results presented here can probably be extended to the $n$-dimensional single-input single-output case.

Acknowledgement-I thank the anonymous referees for several helpful suggestions.

\section{REFERENCES}

Mårtensson, B. (1986). Adaptive stabilization of general, multivariable, continuous- or discrete-time system. In Modelling, Identification and Robust Control. NorthHolland, Amsterdam

Morse, A. S. (1983). Recent Problems in Parameter Adaptive Control. In I. D. Landau (Ed.) Ontils et Modelès Mathématiques pour I'Automatique, l'Analyse de Systèmes et le Traitement du Signal. 733-740.

Mudgett, D. R. and A. S. Morse (1985). Adaptive stabilization of a discrete linear system with an unknown high-frequency gain. IEEE Trans. Aut. Control, AC-30, 798-799.

Nussbaum, R. D. (1983). Some remarks on a conjecture in parameter adaptive control. Syst. Control Lett., 3, 243-246.

Wang, K. and L. Ljung (1989). A discussion of adaptive stabilization and robust adaptive control. Syst. Control Lett., 12, 53-56.

Witlems, J. C. and C. I. Byrnes (1984). Global adaptive stabilization in the absence of information on the sign of the high frequency gain. In Lecture Notes on Control Information Science. Vol. 62, Springer, Berlin. 